\title{
A NOTE ON STRONG JORDAN SEPARATION
}

\author{
JEAN-FranÇOIS LAFONT
}

Abstract

We establish a strengthening of Jordan separation, to the setting of maps $f: X \rightarrow S^{n+1}$, where $X$ is not necessarily a manifold, and $f$ is not necessarily injective.

\section{Introduction}

In a previous paper $[\mathbf{L}]$, we established a result which we termed strong Jordan separation. This was a version of Jordan separation which applied to maps $f: S^{n} \rightarrow S^{n+1}$ which are not assumed to be injective. Under some mild hypothesis, one could nevertheless ensure that the image separated $S^{n+1}$, and that any continuous extension $F: \mathbb{D}^{n+1} \rightarrow$ $S^{n+1}$ surjects onto one of the connected components of $S^{n+1}-f\left(S^{n}\right)$. Recently, Iwaniec and Onninen [IO] found applications of this result in the field of quasi-conformal hyperelasticity. In this short note, we extend our result from $[\mathbf{L}]$ to the broadest possible setting, by establishing the following two results:

Theorem 1. Let $X$ be a compact topological space, $f: X \rightarrow S^{n+1} a$ continuous map, and $U \subset X$ an open subset homeomorphic to an open $n$-disk $\mathbb{D}_{\circ}^{n}$. Assume that

- the map $f: X \rightarrow S^{n+1}$ contains $U$ in its set of injectivity (i.e. $U \subset$ $\left.\operatorname{Inj}(f):=\left\{x \in X \mid f^{-1}(f(x))=x\right\}\right)$, and

- the map $\check{H}^{n}\left(X ; \mathbb{Z}_{2}\right) \rightarrow \check{H}^{n}\left(X-U ; \mathbb{Z}_{2}\right)$ on $\check{C}$ ech cohomology groups induced by the inclusion $X-U \hookrightarrow X$ has a non-trivial kernel.

Then $f(X)$ separates $S^{n+1}$ into at least two connected components. Furthermore, there are precisely two connected components $V_{1}, V_{2}$ of $S^{n+1}-$ $f(X)$ having the property that their closure $\bar{V}_{i}$ intersects $f(U)$. In fact, for these two connected components, we have containments $f(U) \subset \bar{V}_{i}$.

2000 Mathematics Subject Classification. Primary: 57N99; Secondary: 55M05, $57 \mathrm{~N} 45$.

Key words. Jordan separation, Alexander duality, codimension one taming. 
In the previous theorem, one should think of the sets $V_{1}, V_{2}$ as corresponding locally to the two "sides" of $f(U) \cong \mathbb{D}_{\circ}^{n}$ in the ambient $S^{n+1}$.

Theorem 2. Under the hypotheses of the previous theorem, let us further assume that $X$ is a closed subspace of an ambient topological space $\hat{X}$. Define two subgroups of $H_{n}\left(X ; \mathbb{Z}_{2}\right)$ by:

- $K=\operatorname{ker}\left(H_{n}\left(X ; \mathbb{Z}_{2}\right) \rightarrow H_{n}\left(\hat{X} ; \mathbb{Z}_{2}\right)\right)$, and

- $J=\operatorname{im}\left(H_{n}\left(X-U ; \mathbb{Z}_{2}\right) \rightarrow H_{n}\left(X ; \mathbb{Z}_{2}\right)\right)$,

where both maps are induced by the corresponding inclusions of spaces. If $K \nsubseteq J$, then we have that for any continuous extension $F: \hat{X} \rightarrow S^{n+1}$, $F$ surjects onto one of the two open components $V_{i}$.

We will prove both these theorems in Section 2 of our paper. Observe that, other than the condition requiring $U$ to lie in $\operatorname{Inj}(f)$, the hypotheses in both theorems are internal, in the sense that they are statements purely about the (homology of the) spaces $U, X, \hat{X}$, and do not involve the map $f$. In Section 3, we will provide examples showing that the hypotheses of the theorems are necessary. We will also exhibit examples of triples $(\hat{X}, X, U)$ satisfying the homological hypotheses of both theorems.

\section{Acknowledgments}

Tadeusz Iwaniec asked whether the author's arguments for the strong Jordan separation theorem in $[\mathbf{L}]$ could be extended to the case where the source space is a manifold other than the sphere. The present paper was motivated by, and answers, Iwaniec's question.

This research was partially supported by the NSF, under grant DMS0606002, and by an Alfred P. Sloan research fellowship.

\section{Proofs}

Throughout this section, all homology and cohomology groups are understood to have coefficients in $\mathbb{Z}_{2}$. The proofs of both theorems follow closely the proof of the strong Jordan separation in [L, Section 2].

Proof of Theorem 1: We start by recalling that Alexander duality provides us with an isomorphism:

$$
\tilde{H}_{0}\left(S^{n+1}-f(X)\right) \cong \check{H}^{n}(f(X))
$$

hence to show that $f(X)$ separates $S^{n+1}$ it is sufficient to show that $\check{H}^{n}(f(X)) \neq 0$. Now consider the decomposition of $X$ into two open sets, one obtained by shrinking $U$ slightly, the other obtained by enlarging 
$X-U$ slightly. The intersection of these two open sets is an open subset homeomorphic to $S^{n-1} \times \mathbb{R}$. Corresponding to this decomposition, we have an image decomposition of $f(X)$ into two open sets (recall that $U \subset$ $\operatorname{Inj}(f))$. In particular, we can apply the Mayer-Vietoris sequence in Čech cohomology to compute the cohomology of $f(X)$ :

$$
\begin{aligned}
& \cdots \rightarrow \check{H}^{n-1}\left(S^{n-1}\right) \rightarrow \check{H}^{n}(f(X)) \rightarrow \check{H}^{n}(f(U)) \\
& \oplus \check{H}^{n}(f(X-U)) \rightarrow \check{H}^{n}\left(S^{n-1}\right) \rightarrow \cdots
\end{aligned}
$$

Putting in the known terms into the sequence above, we obtain:

$$
\cdots \rightarrow \mathbb{Z}_{2} \rightarrow \check{H}^{n}(f(X)) \rightarrow \check{H}^{n}(f(X-U)) \rightarrow 0
$$

so to show that $\check{H}^{n}(f(X)) \neq 0$, it is sufficient to show that the $\mathbb{Z}_{2} \cong$ $\check{H}^{n-1}\left(S^{n-1}\right)$ injects into $\check{H}^{n}(f(X))$. In order to show this, we compare the Mayer-Vietoris sequence above with the corresponding MayerVietoris sequence for the decomposition of $X$. This gives us the following commutative diagram:

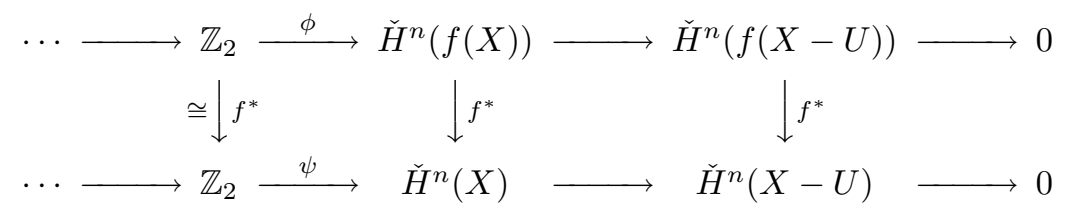

Since we assumed that the map $\check{H}^{n}(X) \rightarrow \check{H}^{n}(X-U)$ has non-trivial kernel, we see that $\psi$ is injective. This forces the composite $\psi \circ f^{*}=f^{*} \circ \phi$ to be injective, and hence the map $\phi$ to be injective, as desired. This allows us to conclude that $0 \neq \check{H}^{n}(f(X)) \cong \tilde{H}_{0}\left(S^{n+1}-f(X)\right)$, and we see that $f(X)$ separates $S^{n+1}$.

Now, the remainder of the proof is virtually identical to that given in $[\mathbf{L}]$. We will briefly sketch out the arguments, referring the reader to [L, Section 2.1] for more details.

To see that there are precisely two connected components $V_{1}, V_{2}$ whose closure intersects $f(U)$, one considers the inclusion $S^{n+1}-f(X) \hookrightarrow$ $S^{n+1}-f(X-U)$. The latter space is obtained from the former by "adding in $f(U)$ ", i.e. we have identifications $S^{n+1}-f(X-U)=\left(S^{n+1}-f(X)\right) \cup$ $f(U)$. By applying Alexander duality to the exact sequence in (1), we obtain:

$$
0 \rightarrow \mathbb{Z}_{2} \rightarrow \tilde{H}_{0}\left(S^{n+1}-f(X)\right) \rightarrow \tilde{H}_{0}\left(\left(S^{n+1}-f(X)\right) \cup f(U)\right) \rightarrow 0 .
$$

This is a homological version of the statement "there are precisely two components of $S^{n+1}-f(X)$ which are incident to $f(U)$ ". 
In order to obtain the statement we desire, we make use of the following elementary result from point set topology: if $\left\{V_{i}\right\}$ is a collection of pairwise disjoint open sets in $\mathbb{R}^{n+1}$, and $Z$ is a connected set which intersects the closure of each $V_{i}$, then $Z \cup\left(\cup V_{i}\right)$ is connected. Now apply this to the situation where the $\left\{V_{i}\right\}$ are the connected components of $S^{n+1}-f(X)$ whose closure intersects $f(U)$, and $Z=f(U)$. For any distinct pair of connected components of $S^{n+1}-f(X)$ whose closure intersects $f(U)$, we will get a corresponding element in $\tilde{H}_{0}\left(S^{n+1}-f(X)\right)$ which maps to zero in $H_{0}\left(S^{n+1}-f(X-U)\right)$. In other words, the rank of the kernel is one less than the number of connected components of $S^{n+1}-f(X)$ whose closure intersects $f(U)$. Since we know that the kernel has rank one, we conclude that there are precisely two connected components $V_{1}, V_{2}$ of $S^{n+1}-f(X)$ whose closure intersects $f(U)$.

Finally, to see that $f(U) \subset \bar{V}_{i}$, take $p \in f(U)$ arbitrary, consider a shrinking sequence of open metric balls $\left\{U_{i}\right\}$ centered at $f^{-1}(p)$. We can apply the same argument as in the previous paragraph, but replacing $f(U)$ by $f\left(U_{i}\right)$. Observe that $f(X-U) \subset f\left(X-U_{i}\right)$ induces an isomorphism on all the Čech cohomology groups, and so by Alexander duality, the homology groups of the complements are also unchanged. This forces the same two components $V_{1}, V_{2}$ to intersect every $f\left(U_{i}\right)$. Since the sets $f\left(U_{i}\right)$ are shrinking down to $\{p\}$, this immediately gives us that $p$ lies in the closure of both $V_{i}$, completing the proof of Theorem 1.

Proof of Theorem 2: Before starting with the proof of the theorem, let us briefly discuss some general background material. For $p \notin f(X)$, we will consider the homomorphism $f_{*}: H_{n}(X) \rightarrow H_{n}\left(\mathbb{R}^{n+1}-p\right) \cong$ $\mathbb{Z}_{2}$. Note that, since all the groups $H_{n}\left(\mathbb{R}^{n+1}-p\right)$ are isomorphic to $\mathbb{Z}_{2}$, we see that for any $p, q \notin f(X)$, there are canonical identifications between the groups $H_{n}\left(\mathbb{R}^{n+1}-p\right)$ and $H_{n}\left(\mathbb{R}^{n+1}-q\right)$. In particular, it makes sense to talk about elements being "the same" or "different" in the groups $H_{n}\left(\mathbb{R}^{n+1}-p\right)$ and $H_{n}\left(\mathbb{R}^{n+1}-q\right)$. Finally, let us fix some notation. Recall that $U \subset X$ is an open set homeomorphic to an open disc $\mathbb{D}_{\circ}^{n}$, which we identify with the unit disk in $\mathbb{R}^{n}$. Fixing this identification, we now denote by $U(r)(r<1)$ the subset of $U$ which corresponds to the open disk of radius $r$. We will use $O$ to denote the point in $U$ which corresponds to the origin.

Let us now argue by way of contradiction: assume that there exists a continuous extension $F: \hat{X} \rightarrow S^{n+1}$ and points $z_{i} \in V_{i}$ with $z_{i} \notin F(\hat{X})$. By hypothesis, $K \nsubseteq J$, so there exists a homology class $\alpha \in H_{n}(X)$ having the following two properties: 
(1) $\alpha \in \operatorname{ker}\left(H_{n}(X) \rightarrow H_{n}(\hat{X})\right)$, and

(2) $\alpha \notin \operatorname{im}\left(H_{n}(X-U) \rightarrow H_{n}(X)\right)$.

Let us consider the image of the class $\alpha$ in each of the homology groups $H_{n}\left(\mathbb{R}^{n+1}-z_{i}\right) \cong \mathbb{Z}_{2}$. To compute this, observe that since $z_{i} \notin F(\hat{X})$, we can factor the map $f_{*}$ as the composition $F_{*} \circ i_{*}$, where $i: X \rightarrow \hat{X}$ is the inclusion. This forces the containment $\operatorname{ker}\left(i_{*}\right) \subseteq \operatorname{ker}\left(f_{*}\right)$, which combined with property (1) in the choice of $\alpha$, ensures that $\alpha$ maps to zero in each of the homology groups $H_{n}\left(\mathbb{R}^{n+1}-z_{i}\right) \cong \mathbb{Z}_{2}$.

So in order to obtain a contradiction, it is sufficient to show that the class $\alpha$ maps to distinct elements in each of the two homology groups $H_{n}\left(\mathbb{R}^{n+1}-z_{i}\right) \cong \mathbb{Z}_{2}$. This is considerably harder; we will proceed in several steps. We will first replace the map $f$ by another map $g$, obtained by locally perturbing $f$ on the interior of $U$. This new map $g$ will have the following properties:

- $g$ is tame on the interior of $U(1 / 2)$,

- $g \equiv f$ on the complement of $U(3 / 4)$,

- $U \subset \operatorname{Inj}(g)$,

- $f$ is homotopic to $g$ in the complement of the points $z_{1}, z_{2}$.

The construction of $g$ can be done by appealing to the important codimension one taming theorem of Ancel-Cannon $[\mathbf{A C}]$ (when $n \geq 4$ ), Ancel $[\mathbf{A}]$ (when $n=3)$, Bing $[\mathbf{B}]($ when $n=2)$ and Schoenflies (when $n=1$ ). We refer the reader to $[\mathbf{L}$, p. 689] for details on how to accomplish this perturbation.

Since $f \simeq g$ in the complement of the $z_{i}$, it is sufficient to show that $g_{*}$ maps $\alpha$ to distinct elements in $H_{n}\left(\mathbb{R}^{n+1}-z_{i}\right)$. Now for the map $g$, we can again apply Theorem 1 , and see that there are exactly two connected components $V_{1}^{\prime}, V_{2}^{\prime}$ of $S^{n+1}-g(X)$ whose closure intersects $g(U)$. Recall that $O \in U$ is the point corresponding to the origin under the homeomorphism identifying $\mathbb{D}_{\circ}^{n}$ with $U$. We know that there exists a sequence of points $x_{i} \in V_{1}^{\prime}, y_{i} \in V_{2}^{\prime}$, with the property that $\lim \left\{x_{i}\right\}=\lim \left\{y_{i}\right\}=g(O)$. We claim that, for each $i$, the $g_{*}(\alpha)$ form distinct elements in the two groups $H_{n}\left(\mathbb{R}^{n+1}-x_{i}\right)$ and $H_{n}\left(\mathbb{R}^{n+1}-y_{i}\right)$.

To see this, we first note that, if the points $p, q$ are chosen in the same connected component of $\mathbb{R}^{n+1}-g(X)$, then the two homomorphisms $g_{*}: H_{n}(X) \rightarrow H_{n}\left(\mathbb{R}^{n+1}-p\right)$ and $g_{*}: H_{n}(X) \rightarrow H_{n}\left(\mathbb{R}^{n+1}-q\right)$ are identical. In particular, it is enough to show that the images of $g_{*}(\alpha)$ are distinct for a specific index $i$. But recall that the map $g$ is tame on $U(1 / 2)$, and hence there exists a global homeomorphism $\phi: \mathbb{R}^{n+1} \rightarrow \mathbb{R}^{n+1}$ with the property that $\phi \circ g$ maps $U(1 / 2)$ into the standard $\mathbb{R}^{n} \times\{0\} \subset \mathbb{R}^{n+1}$. 
Furthermore, for $i$ sufficiently large, $\phi\left(x_{i}\right)$ (respectively $\left.\phi\left(y_{i}\right)\right)$ are points which are locally immediately above (respectively below) the hyperplane $\mathbb{R}^{n} \times\{0\}$. It is of course sufficient to show that $\phi_{*}\left(g_{*}(\alpha)\right)$ represents distinct elements in the two homology groups $H_{n}\left(\mathbb{R}^{n+1}-\phi\left(x_{i}\right)\right)$ and $H_{n}\left(\mathbb{R}^{n+1}-\phi\left(y_{i}\right)\right)$.

Now consider a cycle representing the homology class $\alpha \in H_{n}(X)$; this is a formal linear sum $\sum \sigma_{k}$ of finitely many maps $\sigma_{k}: \Delta^{n} \rightarrow X$. The cycle we are interested in is the image cycle $\phi_{*}\left(g_{*}(\alpha)\right)$, which is represented by the formal linear sum $\sum \tau_{k}$, where each $\tau_{k}=\phi \circ g \circ \sigma_{k}$. In particular, there exists a point $p \in \phi(g(U(1 / 2)))$ with the property that $p$ lies solely in the image of the interior of the finitely many simplices, and $p$ is a non-singular value of each of the maps $\tau_{k}$. Note that we can join $\phi\left(x_{i}\right)$ to $\phi\left(y_{i}\right)$ by a PL-curve $\eta$ which intersects $(\phi \circ g)(X)$ in a single transverse intersection at the point $p$. There are now two possibilities: either (A) the homology class $\phi_{*}\left(g_{*}(\alpha)\right)$ represents distinct elements in the two homology groups $H_{n}\left(\mathbb{R}^{n+1}-\phi\left(x_{i}\right)\right)$ and $H_{n}\left(\mathbb{R}^{n+1}-\phi\left(y_{i}\right)\right)$, in which case we are done, or (B) from intersection theory, we have that the number of intersection points of $\eta$ with the maps $\tau_{k}$ is even.

So we are left with arguing that possibility (B) does not occur. By way of contradiction, if this was the case, one could subdivide the finitely many simplices which intersect $\eta$, obtaining a new cycle $\sum \tau_{k}^{\prime}$ having the additional property that all the maps $\tau_{k}^{\prime}$ whose image passes through $p$ coincide with a fixed map $\tau: \Delta^{n} \rightarrow \mathbb{R}^{n+1}$. At the cost of further subdividing, we may moreover assume that $\tau$ lies entirely within the image of $g(U)$. Now, since there are an even number of copies of $\tau$ in the cycle $\sum \tau_{k}^{\prime}$, and since we are working with $\mathbb{Z}_{2}$-coefficients, we can remove all occurences of this singular simplex from the cycle, resulting in a new cycle $\sum \tau_{k}^{\prime \prime}$, which still represents $\phi_{*}\left(g_{*}(\alpha)\right)$ and with the property that the image of all the singular simplices avoid the point $p$.

Observe that all the subdivisions of the singular simplices $\tau_{k}$ gives rise to subdivisions of the singular simplices $\sigma_{k}$ (recall that we have $\tau_{k}=\phi \circ g \circ \sigma_{k}$ ), since a subdivision of a singular simplex is actually performed at the level of the source space. So corresponding to the cycle $\sum \tau_{k}^{\prime}$ representing $\phi_{*}\left(g_{*}(\alpha)\right)$, we have a corresponding cycle $\sum \sigma_{k}^{\prime}$ representing the original $\alpha$. Let $\sigma$ be the singular simplex corresponding to $\tau$, and recall that the subdivision was chosen fine enough so that $\tau$ was contained inside the image of $g(U)$. In particular, this forces $\sigma\left(\Delta^{n}\right) \subset U$, which we recall lies in the set of injectivity of the map $g$. This implies that there are no "accidental cancellations" due to distinct singular simplices in the chain $\sum \sigma_{k}^{\prime}$ both getting mapped to $\tau$. Since 
$\tau$ occurred an even number of times in the cycle $\sum \tau_{k}^{\prime}$, we have that $\sigma$ likewise occurs an even number of times in the cycle $\sum \sigma_{k}^{\prime}$. Finally, working with coefficients in $\mathbb{Z}_{2}$ means that we can drop all copies of $\sigma$, obtaining a new cycle $\sum \sigma_{k}^{\prime \prime}$ having the following two properties:

- the cycle $\sum \sigma_{k}^{\prime \prime}$ represents the homology class $\alpha$, and

- the cycle $\sum \sigma_{k}^{\prime \prime}$ has image in $X$ which is disjoint from the point $O \in$ $U$.

But now observe that, since $U$ is homeomorphic to $\mathbb{D}_{0}^{n}$, we have that $X-U$ is a deformation retract of $X-\{O\}$. Applying the deformation retraction to the cycle $\sum \sigma_{k}^{\prime \prime}$, we can now obtain a cycle representing $\alpha$, but whose image is contained inside $X-U$. This forces $\alpha \in \operatorname{im}\left(H_{n}(X-\right.$ $\left.U) \rightarrow H_{n}(X)\right)$, contradicting property (2) in our choice of the class $\alpha$. We conclude that possibility (A) must occur, completing the proof of Theorem 2.

\section{Optimality and examples}

Before discussing some specific examples covered by our two theorems, let us start by giving some simple non-examples:

- Take $X=S^{1} \subset \mathbb{R}^{2}$, and let $f$ be the projection onto the interval $[-1,1] \times\{0\} \subset \mathbb{R}^{2} \subset S^{2}$. The set of injectivity does not contain any open set $U$.

- Take $X \subset \mathbb{R}^{2}$ to be the union of the standard unit circle, along with the interval $[1,2]$ on the $x$-axis. If $f$ is the projection onto the interval $[-1,2] \times\{0\} \subset \mathbb{R}^{2} \subset S^{2}$, then we see that $f$ is injective on $U=(1,2)$, but the inclusion $X-U \hookrightarrow X$ induces an isomorphism on $\breve{H}^{1}$, so has trivial kernel.

In both cases, we see that the conclusion to Theorem 1 fails, i.e. $f(X) \subset$ $S^{2}$ fails to separate. Similarly, for Theorem 2, we can consider the following simple example: let $X=S^{1}$, and $f: S^{1} \hookrightarrow S^{2}$ be the embedding into the equator. Let $\hat{X}=S^{1} \times[0,1]$ be an annulus, with $X \subset \hat{X}$ corresponding to $S^{1} \times\{0\}$. Note that since $X \hookrightarrow \hat{X}$ is a homotopy equivalence, the group $K$ in Theorem 2 is automatically trivial, and hence $K \subset J$ holds. It is also clear that there exist extensions $F: S^{1} \times[0,1] \rightarrow S^{2}$ with the property that $F$ fails to surject onto either hemisphere. These simple non-examples show that the hypotheses in Theorems 1 and 2 are indeed necessary.

We now proceed to give some examples of triples $(\hat{X}, X, U)$ which satisfy the homological conditions of both theorems. In particular, for 
each of the following examples of triples, we have that if $f: X \rightarrow S^{n+1}$ is injective on $U$, then: (1) $f(X)$ automatically separates $S^{n+1},(2)$ there are exactly two connected components $V_{1}, V_{2} \subset S^{n+1}-f(X)$ whose closure contain $f(U)$, and (3) any extension of $f$ to a map $F: \hat{X} \rightarrow S^{n+1}$ surjects onto either $V_{1}$ or $V_{2}$.

\section{Example: manifolds pairs.}

Taking $\hat{X}$ to be a compact $(n+1)$-dimensional manifold with nonempty boundary, let $X$ be the boundary of $\hat{X}$, and let $U$ be any open $n$-disk in $X$. Note that both of the groups $\check{H}^{n}(X)$ and $H_{n}(X)$ are free $\mathbb{Z}_{2}$-modules, generated by the connected components of $X$ (each of which is a closed manifold). It is now immediate that the map $\check{H}^{n}(X) \rightarrow$ $\check{H}^{n}(X-U)$ has non-trivial kernel, as removing $U$ changes one of the connected components of $X$ from a closed manifold to a compact manifold with boundary. Similarly, this forces $J=\operatorname{im}\left(H_{n}(X-U) \rightarrow H_{n}(X)\right)$ to miss elements in $H_{n}(X)$ : an explicit element $\alpha \notin J$ is given by the sum of the fundamental classes of the connected components of $X$. Finally, we note that $\alpha \in K$ is the image of the relative fundamental class $\mu \in H_{n+1}(\hat{X}, X)$ of the manifold with boundary $\hat{X}$. From the longexact sequence of the pair $(\hat{X}, X)$, we immediately see that $\alpha$ maps to zero in $H_{n}(\hat{X})$, and hence $\alpha \in K$. This implies that $K \nsubseteq J$, completing the verification of the homological hypotheses in our two theorems.

For a more concrete example, if $X=K^{2}$ denotes the Klein bottle, and if $f: K^{2} \rightarrow \mathbb{R}^{3}$ is a continuous map which contains an open set $U \subset$ $\operatorname{Inj}(f)$, then the first theorem implies that $f\left(K^{2}\right)$ separates $\mathbb{R}^{3}$ into at least two connected components. Our second theorem tells us that, if $\hat{X}=L^{3}$ is the "solid Klein-bottle", and $F: L^{3} \rightarrow \mathbb{R}^{3}$ is an extension of $f$, then $F$ surjects onto one of the two components $V_{1}, V_{2}$ incident to any prescribed open set $U \subset \operatorname{Inj}(f)$.

The reader might also like to compare the example where $\hat{X}=S^{1} \times$ $[0,1]$ with the non-example discussed at the beginning of this section. The distinction lies of course in the choice of $X$, which in the present example is $\partial \hat{X}=S^{1} \times\{0,1\}$, and in the non-example, consisted solely of $S^{1} \times\{0\}$.

\section{Example: pseudo-manifold pairs.}

Recall that an $n$-dimensional pseudo-manifold is a simplicial complex with the property that every $(n-1)$-dimensional simplex is a face of exactly two $n$-simplices. An $(n+1)$-dimensional pseudo-manifold with boundary is defined to be a simplical pair $(\hat{X}, X)$ with the property 
that: (1) $X$ is an $n$-dimensional pseudo-manifold, (2) every $n$-simplex in $X$ is contained in a unique $(n+1)$-dimensional simplex, and (3) every $n$-simplex in $\hat{X}-X$ is contained in exactly two $(n+1)$-simplices. From the homological viewpoint, the important observation is that the constraint on the codimension one simplices ensures that compact pseudo-manifolds still have a notion of a fundamental class (the sum of all top-dimensional simplices). In particular, the arguments given earlier for manifolds easily extend to the pseudo-manifold case.

The distinction with the manifold situation is that pseudo-manifolds are allowed to be singular, but that the singularities are relatively "small", i.e. codimension at least two. The importance of this class of topological spaces comes from the fact that every complex projective algebraic variety is a pseudo-manifold (as the singularities will have complex codimension $\geq 1$ ). Note that complex projective algebraic varieties can always be triangulated (see $[\mathbf{L W}]$ ). Examples of compact pseudo-manifolds with boundary can be obtained by taking a complex projective variety $V \subset \mathbb{C} P^{n}$, taking a suitable real codimension one, smooth submanifold $M^{2 n-1} \subset \mathbb{C} P^{n}$ which intersects $V$ non-trivially, and cutting $\mathbb{C} P^{n}$ open along $M^{2 n-1}$. The portion of $V$ in any of the connected components of the resulting manifold with boundary will yield an example of a compact pseudo-manifold with boundary.

\section{Example: CW-complexes and universality.}

We now proceed to consider the case where both $X$ and $\hat{X}$ are CW-complexes, and where $U \subset X$ is a top-dimensional open cell $e^{n} \subset X$. In this case, we note that Čech cohomology coincides with singular cohomology. Furthermore, it is easy to see from a Mayer-Vietoris sequence argument that the map $\check{H}^{n}(X) \rightarrow \check{H}^{n}\left(X-e^{n}\right)$ has non-trivial kernel precisely if the attaching map $S^{n-1} \hookrightarrow X^{(n-1)}$ for the $n$-cell $e^{n}$ induces the zero map on $(n-1)$-dimensional cohomology (where $X^{(n-1)} \subset X$ is the $(n-1)$-skeleton of $X)$. As such it is easy to construct CW-complexes satisfying the homological conditions of our first theorem. Similarly, it is easy to extend such an $n$-dimensional CW-complex $X$ to an $(n+1)$-dimensional CW-complex $\hat{X}$ satisfying the homological conditions for our Theorem 2. For example, one extension which always works is the case where $\hat{X}$ is taken to be the cone over $X$. To see this, first observe that $\hat{X}$ is contractible, and hence that $H_{n}(\hat{X})=0$. This forces $K=\operatorname{ker}\left(H_{n}(X) \rightarrow H_{n}(\hat{X})\right)=H_{n}(X)$. So as long as the map $H_{n}(X-u) \rightarrow H_{n}(X)$ is not surjective, the pair $(\hat{X}, X)$ will satisfy the homological conditions of our Theorem 2. But observe that, from the 
fact that $X$ is a CW-complex satisfying the conditions of our Theorem 1, we have that $H^{n-1}(X-U) \rightarrow 0 \in H^{n-1}\left(S^{n-1}\right)$. Since we are working with coefficients in $\mathbb{Z}_{2}$, the Ext term in the universal coefficient theorem automatically vanishes, and we can identify the cohomology groups above as the duals of the corresponding homology group. This forces the cohomological statement above to be equivalent to the dual homological statement: $\mathbb{Z}_{2} \cong H_{n-1}\left(S^{n-1}\right) \rightarrow 0 \in H_{n-1}(X-U)$. The Mayer-Vietoris sequence now yields:

$$
H_{n}(X-u) \rightarrow H_{n}(X) \rightarrow \mathbb{Z}_{2} \rightarrow 0
$$

confirming that the first map is not surjective. This completes the verification that taking $\hat{X}$ to be the cone over $X$ always satisfies the homological conditions for Theorem 2 .

Let us conclude with an observation: the example of pseudo-manifolds is, in some sense, a "universal example" amongst CW-complexes. Indeed, let us illustrate what we mean by reconsidering the situation where $(\hat{X}, X)$ are $\mathrm{CW}$-complexes, and $U \subset X$ is the interior of an $n$-cell in $X$. Since $X$ is assumed to satisfy the hypotheses of Theorem 1, the analysis in the previous paragraph gives rise to a homology class $\alpha \in H_{n}(X)$ which is not in the image of $H_{n}(X-U)$. Now recall that, given a homology class $\alpha \in H_{n}\left(X ; \mathbb{Z}_{2}\right)$, in an arbitrary topological space $X$, there exists an $n$-dimensional pseudo-manifold $Y$ and a continuous map $\phi: Y \rightarrow X$ with the property that $\phi_{*}[Y]=\alpha$.

Now it is easy to see, from the constraints on $\alpha$, that the image $\phi(Y)$ must pass through $U$. One can further modify $Y$ so as to ensure that the corresponding $\phi: Y \rightarrow X$ has the property that $\phi: \phi^{-1}(U) \rightarrow U$ is a homeomorphism (this can be done by an argument similar to the one at the end of Section 2). This discussion establishes the following:

Lemma (Universality of pseudo-manifold example). Let $X$ be an $n$-dimensional $C W$-complex, $U$ an $n$-cell in $X$, such that the pair $(X, U)$ satisfies the hypotheses of Theorem 1 for the map $f: X \rightarrow S^{n+1}$. Then there exists a pseudo-manifold pair $\left(Y, U^{\prime}\right)$ and a map $\phi:\left(Y, U^{\prime}\right) \rightarrow(X, U)$, with the property that the pair $\left(Y, U^{\prime}\right)$ satisfies the hypotheses of Theorem 1 for the composite map $f \circ \phi: Y \rightarrow S^{n+1}$.

In particular, the image of $\phi(Y)$ under $f$ already separates $S^{n+1}$, showing that Theorem 1 for $\mathrm{CW}$-complexes is actually a consequence of Theorem 1 for pseudo-manifolds. A similar analysis can be used to show that Theorem 2 for $\mathrm{CW}$-complex pairs can also be deduced from the pseudo-manifold case; we leave the details to the interested reader. 


\section{Example: spaces which are not CW-complexes.}

Finally, we give an example which cannot be deduced from the pseudomanifold case: take $X$ to be the closed topologists sine curve. It is well known that the first singular cohomology group is $H^{1}(X)=0$, while the first Čech cohomology group is $\check{H}^{1}(X) \cong \mathbb{Z}_{2}$. If $U \subset X$ is an open interval in the "sine portion" of $X$, then one can readily verify that both $H^{1}(X-U)=0$ and $\check{H}^{1}(X-U)=0$. In particular, we see that the pair $(X, U)$ satisfy the homological conditions for our Theorem 1, so any continuous map $f: X \rightarrow S^{2}$ which is injective on $U$ will have image that separates. Of course, the fact that $H_{1}(X) \cong H^{1}(X)=0$ tells us that there is no chance of using pseudo-manifolds to detect separation (in contrast to the situation with $\mathrm{CW}$-complexes). For $\hat{X}$, one can again take the cone over $X$; it is easy to verify that the homological conditions for Theorem 2 are indeed satisfied.

\section{References}

[A] F. D. ANCEL, Resolving wild embeddings of codimension-one manifolds in manifolds of dimensions greater than 3, Special volume in honor of R. H. Bing (1914-1986), Topology Appl. 24(1-3) (1986), 13-40.

[AC] F. D. ANCEL AND J. W. CANnon, The locally flat approximation of cell-like embedding relations, Ann. of Math. (2) 109(1) (1979), $61-86$.

[B] R. H. Bing, Approximating surfaces with polyhedral ones, Ann. of Math. (2) 65 (1957), 465-483.

[IO] T. Iwaniec And J. Onninen, Deformations of finite conformal energy: existence, and removability of singularities, Proc. London Math. Soc. (to appear).

[L] J.-F. LAFOnT, Strong Jordan separation and applications to rigidity, J. London Math. Soc. (2) 73(3) (2006), 681-700.

[LW] S. Lefschetz and J. H. C. Whitehead, On analytical complexes, Trans. Amer. Math. Soc. 35(2) (1933), 510-517.

Department of Mathematics

Ohio State University

Columbus, OH 43210

USA

E-mail address: jlafont@math.ohio-state.edu

Rebut el 2 d'agost de 2008. 